Voix et Images

voixetimages

\title{
Attention, oeuvres en cours (Daniel Gagnon et François Gravel)
}

\section{Pierre Hébert}

Volume 16, numéro 2 (47), hiver 1991

Jovette Marchessault

URI : https://id.erudit.org/iderudit/200906ar

DOI : https://doi.org/10.7202/200906ar

Aller au sommaire du numéro

\section{Éditeur(s)}

Université du Québec à Montréal

\section{ISSN}

0318-9201 (imprimé)

1705-933X (numérique)

Découvrir la revue

\section{Citer cet article}

Hébert, P. (1991). Attention, oeuvres en cours (Daniel Gagnon et François

Gravel). Voix et Images, 16(2), 336-340. https://doi.org/10.7202/200906ar d'utilisation que vous pouvez consulter en ligne.

https://apropos.erudit.org/fr/usagers/politique-dutilisation/ 
336 VOIX \& IMAGES / 47, hiver 1991

Roman

\section{Attention, œuvres en cours (Daniel Gagnon et François Gravel)}

par Pierre Hébert, Université de Sherbrooke

Il est difficile d'imaginer deux auteurs plus différents. Daniel Gagnon, après avoir écrit des romans proviocateurs qui ont offensé le lecteur bien pensant, s'est permis une pause de sept années pour 
ensuite produire, régulièrement depuis 1985, des récits déchirés, douloureux, où les thèmes de la mort et de l'amour jouent un rôle dominant. Quant à François Gravel, il aborde, dans ses quatre romans parus depuis 1985, des questions inscrites dans les préoccupations du temps, l'institution scolaire en particulier, au moyen d'un style souvent teinté d'ironie. Un point commun cependant: dans un cas comme dans l'autre, ces deux œuvres en cours - dont la plus récente production - méritent une attention toute particulière.

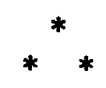

Daniel Gagnon a publié un premier groupe de romans, entre 1972 et 1978 , qui n'ont pas laissé la critique indifférente. Surtout à cause des viandes et Loulou ${ }^{1}$ réussissent ce tour de force enviable de provoquer l'unanimité de la critique contre son ouvre 2 . On ne peut dire que l'entrée de Daniel Gagnon dans la République des lettres s'est faite par la voie facile: personne n'a déroulé le tapis rouge devant cet auteur qui voulait faire croire qu'il se complaît dans la merde, dans le vomi, le sang, le sperme et la morve ${ }^{3}$.

La deuxième série de textes de Daniel Gagnon a reçu, jusqu'à présent, un accueil beaucoup plus favorable; et ce n'est pas que l'auteur se soit assagi, bien au contraire. Mais qu'est-ce qui, cette fois, a davantage plu aux critiques mais, avant tout, aux lecteurs? Une nouvelle thématique? Une écriture lyrique, presque incantatoire? Une « manière " Daniel Gagnon?

Je me souviens, pour ma part, avoir traversé d'un seul élan de lecture la Fille à marier ${ }^{4}$ et avoir été envoûté par cette lettre imaginaire, lyrique, adressée par une jeune fille de douze ans. En une quarantaine de courts chapitres, Jeanne raconte son drame, sa solitude, son délire, mais, surtout la mort de son amoureux Nicolas. Ces lettres désespérées, ces bouteilles jetées à la mer, avaient l'urgence d'une belle et profonde tristesse.

Les œuvres suivantes se sont ensuite montrées à la hauteur des attentes créées. Relisons en particulier la Fée calcinée et ô ma source ! ${ }^{5}$. Le premier de ces récits donne la parole à une morte dont la voix d'outre-tombe engendre des résonances troublantes: je ne crie pas mais cela ne veut pas dire que je ne crie pas, je hurle de douleur en dedans de moi sans fin. (p. 63) Quant à Ô ma source!, récit déroutant sur le plan formel et fait d'un échange de voix et de lettres, il raconte l'histoire d'un pasteur qui trouve asile et amour chez les prostituées.

Il y a un style, une manière Daniel Gagnon qui traverse les œuvres les plus récentes: une thématique marquée par la mort et la recherche de la pureté dans les rapports humains, une presque 
absence de récit et, enfin, une structure narrative fondée sur l'échange dialogique. Son plus récent texte, Venite a cantare ${ }^{6}$, regroupe toutes ces caractéristiques.

Le récit est bref et, dès les premières pages, nous met en présence de la mort. Iolanda Marconi, cantatrice italienne, souffrant du cancer, a été accueillie, au hasard d'une rencontre par Paolo et Francesca qui lui ont fait découvrir, in extremis, l'amitié et l'amour. Or, Iolanda, qui avait rêvé de gloire et d'argent, est également sous le coup d'une poursuite par l'impôt. Le Président de la république, cependant, l'exhorte à revenir chanter pour son peuple et, pour ce, il est prêt à lever ces poursuites. Pourquoi implore-t-il Iolanda à ce point? C'est que la république va mal, elle s'endette, le peuple est fatigué, il a besoin de se distraire (p. 15). Tentative bien inutile, toutefois, parce qu'elle va contre la volonté de Iolanda et que, de toute façon, la Faucheuse fera son œuvre.

Que l'on ne cherche cependant pas ici une histoire, mais plutôt une situation narrativisée. Celle-ci se construit par un jeu d'échanges, de voix qui se démarquent les unes des autres sur un plan que je qualifierais de moral: Iolanda, qui découvre un sens nouveau à la vie, Francesca, qui donne la réplique au Président, et ce dernier, en Créon moderne, qui voit que le peuple a, tout de même, besoin de l'art pour vivre. Pourquoi fallait-il qu'il attende la mort de l'artiste pour s'en apercevoir?

Mais d'où vient l'intérêt de Venite a cantare, de ces chants désespérés qui sont d'immortels sanglots? C'est sans doute que ce style, ces accents qui tiennent de la parole et du chant, touchent le lecteur ou la lectrice dans ce qu'ils ont de plus intime: leur propre mort et, en attendant, la soif de relations humaines plus authentiques. De plus, les personnages de Daniel Gagnon vivent souvent leur accomplissement en dehors des lieux sociaux habituels et leurs longs discours traduisent cette position de rupture, d'éloignement, assortie, malgré tout, d'une recherche de communication. Lyrisme, mort, marginalité du personnage et en particulier de l'artiste: le romantisme est mort, vive le néo-romantisme!

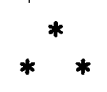

Les romans qu'a signés François Gravel sont-ils plus connus que ceux de Daniel Gagnon? Peut-être, mais cela ne constitue en rien un jugement de valeur; c'est que ce premier s'adresse à un public lecteur beaucoup plus large, si bien que le dernier titre, Bonheur fou ${ }^{7}$, s'est tenu en tête des ventes plusieurs mois après sa parution.

Pourtant, ce ne sont pas là des romans inoffensifs, bien au contraire! N'épargnant aucune de nos institutions contemporaines, 
François Gravel semble cependant éprouver un plaisir particulier à s'attaquer à l'institution scolaire, de même qu'aux modes du temps. La Note de passage, par exemple, qui fut reçu avec enthousiasme, dissèque la figure de l'intellectuel, du professeur en l'occurrence, tout en se moquant des fausses idéologies, avec une ironie caustique. L'Effet Summerhill, pour sa part, entre dans toutes les contradictions de la liberté et de la coercition dans l'éducation.

Mais ce qui intéresse particulièrement ici, c'est le recul, cette saine distance favorisée par l'ironie, et qui fait en sorte que l'auteur nous montre ce qu'il veut dire tout en conservant lui-même un certain détachement. L'effet est bénéfique: le lecteur demeure libre, face à ces textes, de les traiter comme il lui plaît.

Revenons à Bonheur fou: voilà de prime abord un titre étonnant. On se doute bien qu'il n'est pas innocent, que cette collision du bonheur et de la folie n'est pas fortuite. Le personnage principal, Bernard Dansereau, a toujours été préoccupé par la question du bonheur au point de vouloir en trouver le secret. Après des études en médecine et d'autres événements qui l'ont éloigné de cette préoccupation, il se retrouve à travailler à l'hôpital Saint-Jean-de-Dieu. Renouant avec ses questions et convaincu que le bonheur (ou l'aptitude au bonheur) réside quelque part dans le cerveau, il se livrera à diverses experiences touchant tant les cerveaux de poule que les fougères pour en arriver à sa découverte, une méthode magnétique permettant à ses patients de retrouver sinon le bonheur, du moins la bonne humeur.

Le bonheur n'a pas d'histoire, certes, dans le sens le plus restreint d'histoire: au risque d'une réitération sans intérêt, un récit heureux n'est pas possible. Mais un récit sur le bonheur, oui, voilà qui se peut, même si pareille entreprise n'a pas fréquemment cours. Qui, en littérature québécoise, s'est interrogé sur le bonheur? Jacques Godbout, peut-être plus que d'autres. Dans Salut Galarneau!, le héros éponyme, dans son stand de hot-dogs, interroge les gens sur le bonheur; quant à Gregory Francœur, d'Une histoire américaine, il est en Californie pour diriger une enquête sur le bonheur. On pensera également à Jacques Poulin qui, ici et là dans les Grandes Marées ou Volkswagen Blues, revient à la question essentielle du bonheur.

Mais un roman entier où un personnage cherche le secret du bonheur, voilà quelque chose d'exceptionnel, défi que François Gravel relève brillamment. Le sujet convenait parfaitement à ce maître de l'ironie toute en demi-teinte, car, dans cette recherche des clefs du bonheur, l'occasion était belle pour décocher quelques flèches en direction de l'institution médicale et scientifique. À cet égard, que le

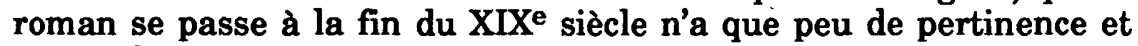
n'affaiblit en rien son efficacité corrosive. 
Le bonheur peut-il s'acquérir par l'étude ou par l'habitude (ou par toute autre force qu'on voudra), ou bien est-ce en vertu d'une forme divine ou encore par un effet de la chance qu'il nous échoit? Voilà la question que se posait Aristote dans Éthique à Nicomaque. François Gravel n'ambitionne nullement de répondre à cette question, encore qu'il l'envisage sous toutes ses formes; et quant à nous, lisons Bonheur fou... pour le simple bonheur de le faire.

1 Daniel Gagnon, Surtout à cause des viandes, Montréal, CLF, 1972, 111 p.; Loulou, Montréal, CLF/Pierre Tisseyre, 1976, $160 \mathrm{p}$.

2 Yvon Boucher, * Daniel Gagnon ou le refus du culturel \#, le Devoir, 10 septembre 1977 , p. 16.

3 Jean-Claude Trait, "Surtout “à cause" du style ", le Devoir, 20 janvier 1973, p. C-3. Jean-Claude Trait ne manque cependant pas de trouver des qualités au premier roman de Gagnon.

4 Daniel Gagnon, la Fille à marier, Montréal, Leméac, $1985,110 \mathrm{p}$.

5 Id., la Fée calcinée, Montréal, VLB éditeur, 1987, 115 p.; Ố ma sourcel, Montréal, Guérin, 1988, 191 p.

6 Id., Venite a cantare, Montréal, Leméac, 1990, 73 p. Notons que Daniel Gagnon vient aussi de faire paraitre un recueil de nouvelles, Circumnavigatrice, Montréal, XYZ éditeur, 1990, 99 p.

7 François Gravel, Bonheur fou, Montréal, Boréal, 1990, 302 p. 